\title{
Deterministic-aided single dataset STAP method based on sparse recovery in heterogeneous clutter environments
}

\author{
Wei Wang ${ }^{1,2^{*}}$, Lin Zou', Xuegang Wang ${ }^{1}$ and Yang Yang ${ }^{2}$
}

\begin{abstract}
Traditional space-time adaptive processing (STAP) usually needs many independent and identically distributed (i.i.d) training datasets for estimating clutter covariance matrix (CCM). But this requirement is hardly satisfied in the heterogeneous clutter environments, which lead to an inaccurate estimation of CCM and accordingly degrade the performance of STAP significantly. To improve the performance of STAP in heterogeneous environments, a novel deterministic-aided (DA) single dataset STAP method based on sparse recovery technique (SR) is proposed in this paper. This presented algorithm exploits the property that the clutter components of side-looking airborne or spaceborne radar are distributed along the clutter ridge to estimate the CCM of the cell under test (CUT) without any secondary training data. The new method only uses a single CUT data to acquire a high-resolution angle-Doppler power spectrum using sparse recovery (SR) approach and then employs a new adaptive deterministic-aided generalized inner product (GIP) algorithm to recognize and select the clutter components in the CUT angle-Doppler power spectrum automatically. Subsequently, the CCM, which is used to construct the weights of STAP filter, can be effectively estimated by the selected clutter components to fulfill the final STAP filter processing. Simulation results verify the effectiveness of the proposed detection method.
\end{abstract}

Keywords: Space-time adaptive processing (STAP), Deterministic-aided (DA), Sparse recovery (SR), Generalized inner product (GIP), Heterogeneous clutter

\section{Introduction}

Space-time adaptive processing (STAP), a twodimensional space-time adaptive filtering operation, is an effective and important technique which is widely used in airborne or spaceborne radar for detecting moving target in strong clutter background [1-4]. And in most STAP applications, the training data in the neighborhood of the range cell of interest are employed to estimate the clutter covariance matrix (CCM) which is used to compute the filter's weights adaptively $[5,6]$. Thus, to guarantee the accuracy of the estimation of CCM, two major conditions should be satisfied. One is the training data from adjacent range cells should be target-free and contain clutters with the same statistic

\footnotetext{
* Correspondence: 415382448@qq.com

${ }^{1}$ University of Electronic Science and Technology of China, Chengdu 611731, China

${ }^{2}$ China Aerodynamics Research and Development Center, Mianyang 621000, China
}

characteristics as the cell under test (CUT), which is referred to as independent and identically distributed (i.i. d) condition [4]. The other, according to RMB rule [4], is the number of training data snapshots should be larger than twice the number of system degrees of freedom (DOFs) to achieve the output signal-to-interferenceplus-noise ratio (SINR) loss is $<3 \mathrm{~dB}$. Unfortunately, most of the actual scenarios, which the airborne or spaceborne radar faces onto, are non-stationary and include heterogeneous clutters or a high target density. In this case, so many i.i.d and target-free training samples are difficult to be obtained. Therefore, it results in a false estimation of CCM, which leads to a performance decrease of clutter suppression or even a self-nulling of target signal.

So many reduced-dimension or reduced-rank methods [7-10] have been proposed to overcome the aforementioned problem. However, the number of necessary training samples mentioned in such approaches may still 
be large when facing a severe heterogeneous environment. Moreover, several non-homogeneity detection (NHD) algorithms [11-15] have been applied in the heterogeneous environments, such as the power-selected training (PST) algorithm [11] and the generalized inner product (GIP) algorithm [12-15]. They all seek to select the training sample whose CCM is similar to that of the CUT data. However, the PST algorithm may result in target self-nulling and the GIP algorithm would result in an ineffective estimation of CCM if the CUT data is heterogeneous with most of the initial training samples. On the other hand, since some unqualified training samples should be removed, the GIP algorithm cannot achieve an acceptable performance of clutter suppression when the number of training samples is deficient. A knowledge-aided (KA) STAP method, which introduces the benefits of the digital terrain data into STAP applications, was proposed in [16]. Nevertheless, the reflectivity change with grazing angles and the inaccurate registering of the terrain data would degrade its performance. Additionally, the persymmetric structure of the interference covariance matrix and the symmetric property of power spectral density of the clutter can also be used as priori knowledges to improve the detection performances in training-limited scenarios [17-20]. And some STAP detection methods, which allow one to identify the degree of accuracy of the prior knowledge and combine the prior information with the secondary data in an appropriate way, were proposed in $[21,22]$. To further deal with the training-limited problem, a detection scheme using a linear combination of some available a priori models to model the inverse covariance matrix was reported in [23], a newly proposed detection method using two sets of training data are not limited by the conventional constraint on the cardinality of the classic training dataset [24], and a geometric approach to covariance matrix estimation can also achieve considerable SINR improvement in training-starved regimes [25]. Some other related applications to MIMO radar can be found in [26, 27]. Recently, sparse recovery (SR) technique has also been applied to STAP processing [28-32]. The SR-STAP algorithm can accurately estimate CCM or the subspace of clutter with much fewer training samples than the conventional STAP algorithms. As all of the methods aforementioned above still need training samples or assume the training samples are targetfree, they still have some limitations to deal with the serious non-stationary radar scenarios.

We call the STAP algorithm that needs to use training samples to estimate CCM as the two dataset STAP (TDS-STAP) algorithm. Obviously, the clutter suppression performance of TDS-STAP is largely limited by the number and statistic characteristics of the training samples. Furthermore, to lower the limit on the training sample, several single dataset STAP (SDS-STAP) algorithms have been proposed [32-37]. They only use CUT data for CCM estimation or clutter suppression. The direct data domain STAP (D3-STAP) [33-35] and the maximum likelihood estimation detector (MLED) algorithm $[36,37]$ are two typical SDS-STAP algorithms, but their benefits are available at the expense of system DOFs loss. Another direct data domain STAP approach using sparse recovery (D3SR-STAP) was proposed in [32]. It can effectively estimate CCM based on the angleDoppler power spectrum got by sparse recovery technique only with CUT data. Though this method keeps the full system DOFs, the requirement for prior knowledge of the signal of interest (SOI) area limits its effectiveness because the prior knowledge is not easy to be got accurately.

In this paper, we propose a novel deterministic-aided (DA) single dataset (SDS) STAP method based on sparse recovery (SR) technique, which is abbreviated to DASDS-SRSTAP, for side-looking airborne or spaceborne radar in heterogeneous clutter environments. This method needs neither any training data nor any prior knowledge of SOI while suffering no loss of DOFs. We exploit the property that the clutter components of sidelooking airborne or spaceborne radar are distributed along the clutter ridge to fulfill the estimation of CCM only with CUT data. We first calculate the highresolution angle-Doppler power spectrum of CUT data using SR technique. Then, a new adaptive deterministicaided GIP (DA-GIP) algorithm is presented to recognize and select the clutter components automatically in the angle-Doppler power spectrum. Subsequently, the CCM, which is employed to construct the weights of STAP filter, can be estimated by the selected clutter components to fulfill the final STAP filter processing. Simulation results verify the effectiveness of the proposed detection method.

The paper is organized as follows. Section 2 is devoted to the data model of side-looking airborne or spaceborne radar, while the detailed rationale of the new method, including the SR model and the new DA-GIP algorithm, are presented in Section 3. In Section 4, simulations are given to show the effectiveness of the proposed DASDS-SRSTAP method. Conclusions are finally presented in Section 5.

\section{Data model}

Consider a side-looking uniform linear array (ULA) radar platform that consists of $N$ identical antenna elements with an inter-element spacing $d=\lambda / 2$. It receives $M$ pulses with pulse repetition frequency (PRF) $f_{r}$ in a coherent processing interval (CPI) for each range gate. $\lambda$ is the radar wavelength. The simplified coordinate for the side-looking ULA radar platform is shown in Fig. 1. 


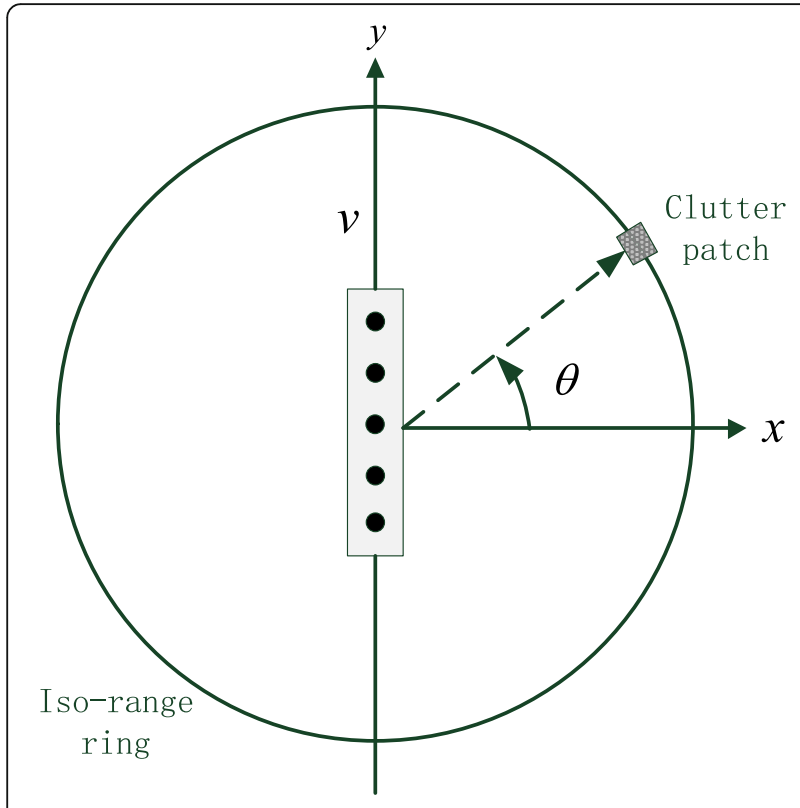

Fig. 1 Simplified side-looking ULA radar platform geometry

Without loss of generality, we neglect the elevation dimension in the coordinate [4]. The velocity of the platform and the azimuth angle are $v$ and $\theta$, respectively. As we only use the primary CUT data, the range gate dimension can be overlooked in the following discussions, and the processing algorithm works independently in each range gate.

For the side-looking radar, $N_{c}$ clutter patches are assumed to be independent with one another and be of uniform distribution in each iso-range ring. Hence, the clutter data can be modeled as the summation of these $N_{c}$ independent clutter patches with different spatial and Doppler frequencies [4]. Assuming no antenna crabbing, the spatial frequency $\phi_{c, i}$ and normalized Doppler frequency $\psi_{c, i}$ of the $i$ th clutter patch can be, respectively, given by

$$
\begin{aligned}
& \phi_{c, i}=d \cos \theta_{c, i} / \lambda \\
& \psi_{c, i}=2 v \cos \theta_{c, i} /\left(\lambda f_{r}\right)
\end{aligned}
$$

where $\theta_{c, i}$ indicates the angle of the $i$ th clutter patch. The space-time steering vector of the $i$ th clutter patch is given by

$$
\mathbf{v}_{i}=\mathbf{v}\left(\psi_{c, i}, \phi_{c, i}\right)=\mathbf{b}\left(\psi_{c, i}\right) \otimes \mathbf{a}\left(\phi_{c, i}\right)
$$

where $\mathbf{v}(\psi, \phi)=\mathbf{b}(\psi) \otimes \mathbf{a}(\phi)$ and $\otimes$ denote the space-time steering vector and the Kronecker product, respectively. $\mathbf{a}\left(\phi_{c, i}\right)$ and $\mathbf{b}\left(\psi_{c, i}\right)$ are the spatial and temporal steering vector of the $i$ th clutter patch, respectively, which are defined by

$$
\begin{aligned}
& \mathbf{a}\left(\phi_{c, i}\right)=\left[1, \exp \left(j 2 \pi \phi_{c, i}\right), \cdots, \exp \left(j(N-1) 2 \pi \phi_{c, i}\right)\right]^{T} \\
& \mathbf{b}\left(\psi_{c, i}\right)=\left[1, \exp \left(j 2 \pi \psi_{c, i}\right), \cdots, \exp \left(j(M-1) 2 \pi \psi_{c, i}\right)\right]^{T}
\end{aligned}
$$

where $[\mathrm{g}]^{T}$ denotes the transpose operation. Then, ignoring the range ambiguities, the total clutter return from a given iso-range ring can be expressed by [4]

$$
\mathbf{c}=\sum_{i=1}^{N_{c}} \sigma_{c, i} \mathbf{v}_{i}
$$

where $\sigma_{c, i}$ denotes the complex amplitude of the $i$ th clutter patch. Moreover, considering the incoherence between each clutter patch, the clutter covariance matrix can be simplified as [4]

$$
\mathbf{R}_{c}=E\left[\mathbf{c c}^{H}\right]=\sum_{i=1}^{N_{c}}\left|\sigma_{c, i}\right|^{2} \mathbf{v}_{i} \mathbf{v}_{i}^{H}
$$

where $E[\mathrm{~g}],[\mathrm{g}]^{H}$, and $|\mathrm{g}|$ denote expectation operation, conjugate transpose, and the modulus of a complex, respectively.

Similarly, the spatial frequency $\phi_{t}$ and normalized Doppler frequency $\psi_{t}$ of the hypothetic target can be, respectively, given by

$$
\begin{aligned}
& \phi_{t}=d \cos \theta_{t} / \lambda \\
& \psi_{t}=2\left(v_{t}-v\right) \cos \theta_{t} /\left(\lambda f_{r}\right)
\end{aligned}
$$

where $\theta_{t}$ and $v_{t}$ denote the angle and velocity of the target, respectively. The target signal can be expressed by

$$
\mathbf{s}=\sigma_{t} \mathbf{v}\left(\psi_{t}, \phi_{t}\right)=\sigma_{t} \mathbf{v}_{t}
$$

where $\mathbf{v}_{t}=\mathbf{v}\left(\psi_{t}, \phi_{t}\right)=\mathbf{b}\left(\psi_{t}\right) \otimes \mathbf{a}\left(\phi_{t}\right)$ and $\sigma_{t}$ denote the space-time steering vector and the complex amplitude of the target, respectively.

As is well known, the optimal filter weights of STAP can be given by

$$
\mathbf{w}_{\mathrm{opt}}=\kappa \mathbf{R}_{c}^{-1} \mathbf{v}_{t}
$$

where $\kappa$ is an arbitrary scalar which does not alter the output SINR and $\mathbf{R}_{c}^{-1}$ denotes the inverse matrix of $\mathbf{R}_{c}$. In order to satisfy the constant false alarm rate (CFAR) property, we usually set $\kappa=\left(\mathbf{v}_{t}^{H} \mathbf{R}_{c}^{-1} \mathbf{v}_{t}\right)^{-1 / 2}$ [4]. The filter output power is then compared to a threshold according to the matched filter (MF) test $[2,6]$

$$
y=\left|\mathbf{w}_{o p t}^{H} \mathbf{x}\right|^{2}=\frac{\left|\mathbf{v}_{t}^{H} \mathbf{R}_{c}^{-1} \mathbf{x}\right|^{2}}{\mathbf{v}_{t}^{H} \mathbf{R}_{c}^{-1} \mathbf{v}_{t}}{\underset{H}{H_{1}}}_{\mathcal{S}_{1}} \eta
$$

where $\eta$ is the threshold, $H_{0}$ and $H_{1}$ mean no target and 
a target is present, respectively, and $\mathbf{x}$ is the test data snapshot.

Since $\mathbf{R}_{c}$ is unknown in actual scenarios, the conventional STAP generally employs training samples, which are assumed to be i.i.d and target-free, to estimate CCM. This approach is well known as a TDS-typed algorithm. For instance, using the classical sample matrix inverse (SMI) algorithm [1], CCM can be estimated by

$$
\hat{\mathbf{R}}_{c-\mathrm{SMI}}=\frac{1}{L} \sum_{l=1}^{L} \mathbf{x}(l) \mathbf{x}(l)^{H}
$$

where $\mathbf{x}(l)$ denotes the $l$ th training sample snapshot and $L$ is the number of training samples. Subsequently, $\mathbf{R}_{c}$ in (11) and (12) can be replaced by $\hat{\mathbf{R}}_{c-S M I}$ to compute $\mathbf{w}_{\text {opt }}$ and make MF detection test in practice. Note that if the target-like signals appear in the training samples or the clutter characteristics of the training samples are nonhomogeneous with those of the CUT data, expression (13) will lead to an inaccurate estimation. This would degrade the STAP performance significantly, especially in the case of fewer training samples. In the next section, a novel deterministic-aided single dataset STAP method based on sparse recovery (DA-SDS-SRSTAP) will be described in detail, which can overcome these problems in some extent to improve the detection performance in the heterogeneous radar scenario.

\section{DA-SDS-SRSTAP algorithm}

The proposed algorithm is composed of three primary steps. We first calculate the high-resolution angleDoppler power spectrum using SR technique only with the CUT data. Then, a new adaptive deterministic-aided GIP (DA-GIP) algorithm, based on the property that the clutter components are distributed around the clutter ridge, is proposed to recognize and select the clutter components automatically in the CUT angle-Doppler power spectrum. Subsequently, the CCM, which is employed to form the weights of STAP filter, can be estimated by the selected clutter components to implement the final STAP filter processing. The three steps will be detailedly discussed in the following subsections.

\subsection{Getting CUT angle-Doppler power spectrum using SR} The CUT data snapshot, which includes target and clutter components, can be written as

$$
\mathbf{x}_{0}=\mathbf{s}+\mathbf{c}+\mathbf{n}
$$

where $\mathbf{n}$ is white additive noise. Note that recently developed SR algorithm, which can work on the single dataset case, provides an effective approach for STAP application without the performance degradation caused by DOFs loss, as is the conventional D3-STAP method. SR algorithm discretizes the whole angle-Doppler plane into
$N_{s} \times N_{d}$ bins, where $N_{s}=\rho_{s} N, N_{d}=\rho_{d} M\left(\rho_{s}, \rho_{d}>1\right)$ are the number of the quantization of spatial and Doppler frequency axis, respectively. Then, we reformulate the target and clutter components in the form of sparse representation and the received CUT data snapshot $\mathbf{x}_{0}$ in (14) can be rewritten as [28]

$$
\mathbf{x}_{0}=\boldsymbol{\Phi} \mathbf{\gamma}+\mathbf{n}
$$

where $\Phi$ and $\boldsymbol{\gamma}$ are $M N \times N_{s} N_{d}$ overcomplete space-time steering dictionary and $N_{s} N_{d} \times 1$ angle-Doppler profile vector, respectively, as given by

$$
\begin{aligned}
& \boldsymbol{\Phi}=\left[\mathbf{v}\left(\psi_{1}, \phi_{1}\right), \mathrm{L}, \mathbf{v}\left(\psi_{1}, \phi_{N_{s}}\right), \mathrm{L}, \mathbf{v}\left(\psi_{N_{d}}, \phi_{N_{s}}\right)\right] \\
& \boldsymbol{\gamma}=\left[\gamma_{1}, \gamma_{2}, \mathrm{~L}, \gamma_{N_{s} N_{d}}\right]^{T}
\end{aligned}
$$

where the symbols $\phi_{p},\left(1 \leq p \leq N_{s}\right)$ and $\psi_{q},\left(1 \leq q \leq N_{d}\right)$ denote the quantized spatial and Doppler frequencies respectively. As the theory of SR-STAP [28], a nonzero element from any angle-Doppler grid point, corresponding to a nonzero entry in $\gamma$, would suggest the presence of a scatter at that particular angle and Doppler frequency. Hence, a high-resolution angle-Doppler power spectrum of the CUT data, which shows the information of clutter and target, can be obtained by

$$
\mathbf{P}_{S R}=|\boldsymbol{\gamma}|^{2}
$$

Considering the target and clutter are sparsely distributed in the whole angle-Doppler plane [28], finding the solution of $\gamma$ can be stated as a NP-hard SR problem that represents an interesting signal using the minimum number of vectors from an overcomplete dictionary (set of vectors). Much research effort has been invested in finding some feasible approaches to solve this problem [28-32]. In the following simulations of this work, we just employ, but not limited to, a common SR algorithm named as the focal underdetermined system solver (FOCUSS) algorithm [32] to estimate the CUT angle-Doppler power spectrum. For simplicity, the angle-Doppler power spectrum gained using SR technique is referred to as SR angle-Doppler power spectrum in the followings.

\subsection{DA-GIP algorithm for CCM estimation}

It is noted that, according to expression (1) and (2), there is a linear relationship between the spatial frequency and normalized Doppler frequency of the clutter, which can be rewritten as

$$
\psi_{c, i}=\beta \phi_{c, i}, \beta=2 v / d f_{r} .
$$

Theoretically, this deterministic relationship means that all the clutter components for moving radar platform should be distributed along a line that is well known as clutter ridge in the angle-Doppler domain. $\beta$ 
denotes the slope of clutter ridge, which can be computed using the prior knowledge of platform velocity, the interelement spacing value of antenna array, and the PRF. In other words, once these system parameters are fixed, the clutter ridge will be deterministic. Without loss of generality and to avoid Doppler aliasing, $\beta$ is equivalent to 1 in the following discussions. This deterministic angle-Doppler structure of clutter is illustrated in Fig. 2. It can be observed that the clutter ridge is the diagonal of angle-Doppler plane. Ideally, the nonzero elements located in the clutter ridge can be regarded as clutter components to estimate CCM. However, in practice, because of the impact of inner clutter motion (ICM) or the inaccuracy of prior knowledge, the clutter components will extend to the area adjacent to the ideal clutter ridge, as is shown in the shadow region in Fig. 2 . In this case, if we still use the clutter components just located in the ideal clutter ridge to estimate CCM and apply the result for STAP filter, the clutters cannot be suppressed completely and the residual will further deteriorate the STAP performance severely. Even more unfortunately, the extension, which may be variable for different range bins in the heterogeneous radar scenario, is hard to be predicted in the single dataset condition. Consequently, to cope with the uncertain clutter spread only with the CUT data, we propose a novel DA-GIP algorithm, based on the deterministic property that the clutter components are mainly distributed around the clutter ridge, to recognize and select the whole clutter components in the SR angle-Doppler power spectrum for effective CCM estimation.

The main idea of the DA-GIP algorithm is to find a set of candidate CCM estimation $\left\{\hat{\mathbf{R}}_{c, k}, k=1,2, \mathrm{~L}\right\}$ based on the deterministic knowledge of clutter ridge and then determine which one is the best approximation to the real CCM according to the new defined DA-GIP formulation. Recall that the conventional GIP algorithm,

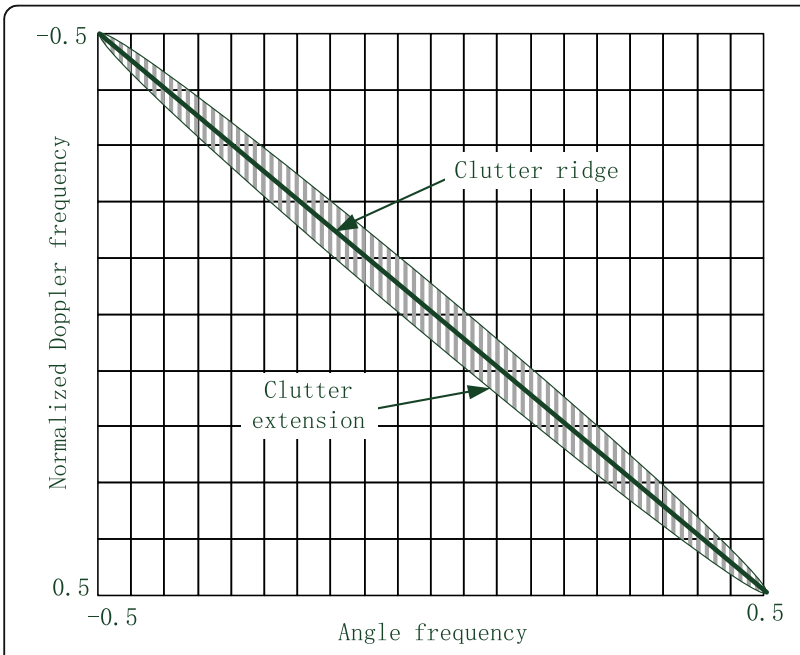

Fig. 2 Angle-Doppler structure of clutter for moving radar platform $(\beta=1)$ which is used for training sample selection, has the following quadratic form $[12,13]$.

$$
Q(l)=\mathbf{x}(l)^{H} \mathbf{R}_{c}^{-1} \mathbf{x}(l)=\left|\mathbf{R}_{c}^{-1 / 2} \mathbf{x}(l)\right|^{2}
$$

where $\mathbf{x}(l)$ and $\mathbf{R}_{c}$ denote the $l$ th training sample snapshot and the true CCM, respectively. Actually, (19) measures the power of training sample whitened by $\mathbf{R}_{c}^{-1 / 2}$. Inspired by the original GIP thought, the DA-GIP formulation for the CUT data is defined by

$$
Q_{0}(k)=\mathbf{x}_{0}{ }^{H} \hat{\mathbf{R}}_{c, k}^{-1} \mathbf{x}_{0}=\left|\hat{\mathbf{R}}_{c, k}^{-1 / 2} \mathbf{x}_{0}\right|^{2}
$$

where $\hat{\mathbf{R}}_{c, k}$ denotes the $k$ th candidate CCM estimation. The value of $Q_{0}(k)$, which is referred to as DA-GIP value, will be used to judge whether its corresponding $\hat{\mathbf{R}}_{c, k}$ is the closest approximation to the real CCM.

Assuming the number of the quantization of spatial frequency axis equals to that of the Doppler frequency axis, i. e., $N_{s}=N_{d}$, the whole procedure can be done as follows.

Step 1: find out some particular nonzero elements of the angle-Doppler profile vector $\gamma$, whose corresponding points in the angle-Doppler plane should satisfy the condition that

$$
p=q \pm(k-1),\left(1 \leq p \leq N_{s}, 1 \leq q \leq N_{d}\right)
$$

where $p$ and $q$ denote the indices of the discretized spatial and Doppler frequency axis, respectively, and $k$ is the iterative time that starts from $k=1$. Then, the indices of these nonzero elements in $\gamma$ are recorded as a set $\Gamma_{k}$. Note that this step is to seek out those clutter components in the clutter ridge at the first iterative time, which can be interpreted in Fig. 3. Those points at the diagonal of the discretized angle-Doppler plane are considered from the beginning.

Step 2: form a new set $\Omega_{k}=\left\{\Gamma_{1}, \mathrm{~L} \Gamma_{k}\right\}$, which is regarded as the $k$ th candidate set of clutter components, and calculate the $k$ th candidate CCM estimation $\hat{\mathbf{R}}_{c, k}$ using the SR expression given by [31].

$$
\hat{\mathbf{R}}_{c, k}=\sum_{h=1}^{H_{k}}\left|\boldsymbol{\gamma}\left(\Omega_{k}(h)\right)\right|^{2}\left(\boldsymbol{\Phi} \mathbf{e}_{\Omega_{k}(h)}\right)\left(\boldsymbol{\Phi} \mathbf{e}_{\Omega_{k}(h)}\right)^{H}
$$

where $H_{k}$ is an integer and denotes the number of elements in set $\Omega_{k}$ and $\mathbf{e}_{\Omega_{k}(h)}$ is a column vector of all zeros except for a one in the position of index $\Omega_{k}(h)$, which can be expressed as

$$
\mathbf{e}_{\Omega_{k}(h)}=\left[0_{1}, \mathrm{~L}, 0_{\Omega_{k}(h)-1}, 1_{\Omega_{k}(h)}, 0_{\Omega_{k}(h)+1}, \mathrm{~L}, 0_{N_{s} N_{d}}\right]^{T}
$$

Step 3: first calculate the DA-GIP value $Q_{0}(k)$, which is rewritten as 


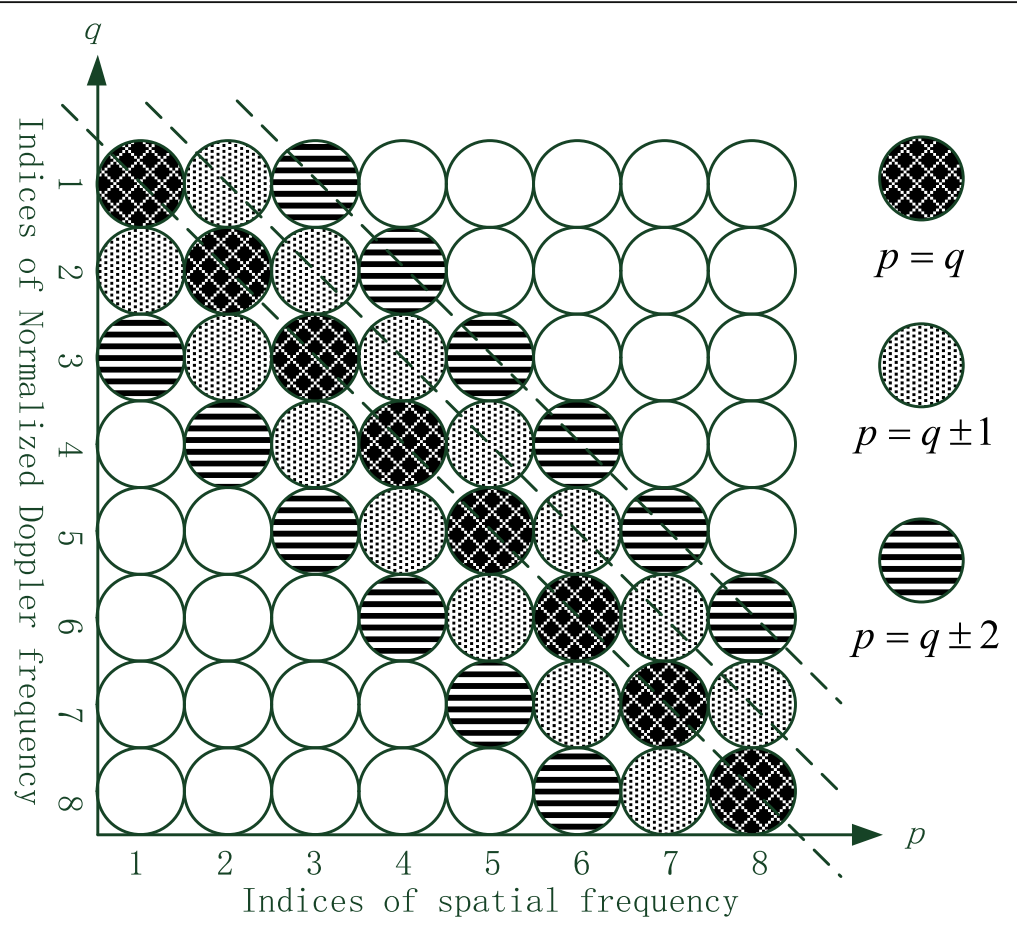

Fig. 3 Illustration of the strategy for clutter component selection

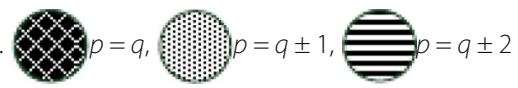

$$
Q_{0}(k)=\mathbf{x}_{0}{ }^{H} \hat{\mathbf{R}}_{c, k}^{-1} \mathbf{x}_{0} .
$$

Then, if $k=1$, set $k=k+1$ and return to step 1 ; otherwise, continue to step 4 .

Step 4: first calculate the error value between $Q_{0}(k)$ and $Q_{0}(k-1)$, which is given by

$$
\Delta_{k}=10 \times \log 10\left(\frac{Q_{0}(k-1)}{Q_{0}(k)}\right), k>1 .
$$

If $\Delta_{k}>\xi$, set $k=k+1$ and return to step 1; otherwise, stop the iteration. $\xi$ is a very small positive real number, which is referred to as error threshold. Denoting $K$ as the last iterative time, then the final available CCM estimation $\hat{\mathbf{R}}_{c-\text { DA-GIP }}$ can be given by

$$
\hat{\mathbf{R}}_{c-\mathrm{DA}-\mathrm{GIP}}=\hat{\mathbf{R}}_{c, K}
$$

The whole procedure can be summarized in Fig. 4 . Though the nonzero elements at the clutter ridge can be regarded as valid clutter components with no doubt at the beginning, the set $\Omega_{1}$ may not include all of the clutter components and $\hat{\mathbf{R}}_{c, 1}$ may not be a good estimation of CCM because the clutter components always spread out of the clutter ridge due to the effect of ICM and knowledge error. Consequently, we should extend the searching area to the outside of the clutter ridge gradually, until the clutter components have been completely selected out. Then, one more grid point on both sides of the clutter ridge in the discretized angle-Doppler plane can be added into the searching areas at each new iterative time, which can be interpreted in Fig. 3. Every extended searching process will give an extra set of clutter components and a new CCM estimation, respectively, as is described in step 2. On the other hand, the DA-GIP value $Q_{0}(k)$ in step 3 is used to measure the accuracy of those candidate CCM estimations. According to expression (21), the DA-GIP value is actually the power of the CUT data whitened by $\hat{\mathbf{R}}_{c, k}^{-1 / 2}$. Remarkably, the CUT data that is whitened with more clutter components should have lower power than that whitened with fewer clutter components. In other words, the DA-GIP value $Q_{0}(k)$ ought to decrease with the increasing of iterative time $k$, until the $K$ th iterative time when $\Omega_{K}$ contains all of the clutter components distributed around the clutter ridge in the SR angle-Doppler power spectrum. Thus, the corresponding CCM estimation $\hat{\mathbf{R}}_{c, K}$ can be taken as the closest approximation to the real CCM. Therefore, when the error of the DA-GIP values $\Delta_{k}$ between two adjacent iterative times becomes very little, it is reasonable to break the iteration and confirm that the CCM has been correctly estimated with full clutter components in the discretized SR angle-Doppler power spectrum, as is described in the step 4 .

Actually, on the assumption that clutter power is usually much larger than noise power, we can set the error 


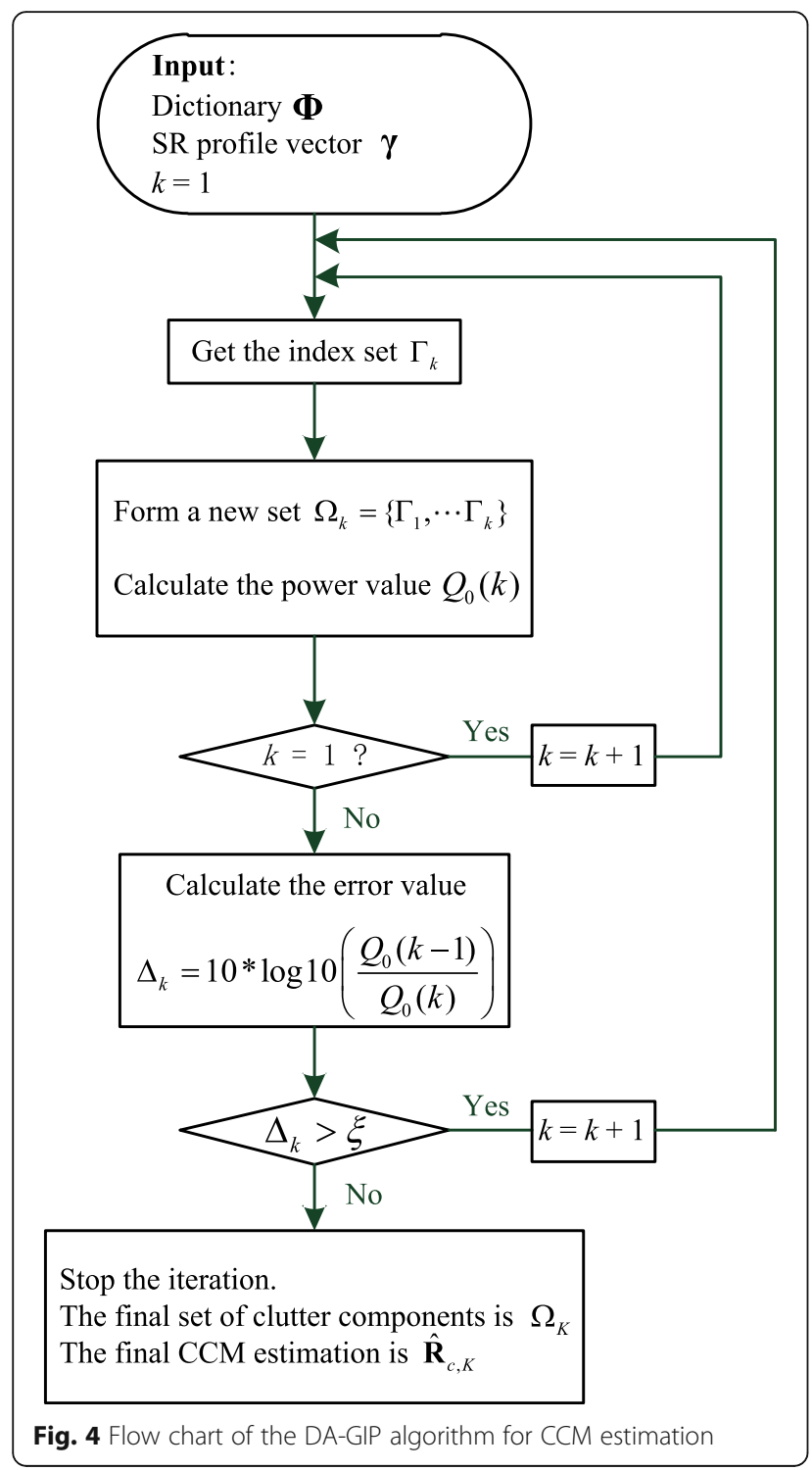

threshold in step 4 depending on noise power. We think that if the whole clutter components have not been included in the data set $\Omega_{k}$ and not been completely whitened, there are still some clutter residuals in the DA-GIP value $Q_{0}(k)$. Moreover, $Q_{0}(k-1)$ should contain more clutter residuals than $Q_{0}(k)$. Therefore, in this case, the error value $\Delta_{k}$ between $Q_{0}(k)$ and $Q_{0}(k-1)$ should also contain the residual clutter components and should be larger than noise power. On the other hand, once all the clutter components have been found out and been completely whitened, the residuals in the DA-GIP value are just noise and target components. After that, considering the target components consisted in the two adjacent DA-GIP values can be mutually canceled out, only the noise component is left in the error value between the two adjacent iterative times. Consequently, it is reasonable to set the error threshold $\xi$ depending on noise power. In practice, it is well known that the noise power can be measured in advance.

Furthermore, the convergence of the comparison in step 4 also depends on the error threshold. According to the analysis above, when the error threshold is equal to or larger than the noise power, the comparison should be convergent. However, if the error threshold is set to be much larger than the noise power, the iteration will stop when the whole clutter components have not been selected out to estimate the CCM, which will result in an inaccurate CCM estimation. Contrarily, when the error threshold is lower than the noise power, the comparison may be not convergent. It is because that although the error value $\Delta_{k}$ may be less than the noise power, it may be still larger than the error threshold which is set to be smaller than the noise power. Consequently, we suggest that the error threshold is best to be equivalent to or slightly larger than the noise power.

\subsection{STAP filter strategy}

Substituting $\hat{\mathbf{R}}_{c \text {-DA-GIP for }} \mathbf{R}_{c}$ in expression (11) and (12), we can implement the final filter processing. Note that the nonzero elements of the SR angle-Doppler power spectrum indicate the angle-Doppler information of the clutter and target, and the whole clutter components have been marked out by the DA-GIP algorithm. Therefore, we just need to test the other nonzero elements, except for those selected clutter ones, for target detection. We denote the spatial and normalized Doppler frequency pairs of those non-selected nonzero elements as $\left\{\left(\phi_{j}^{\prime}, \psi_{j}^{\prime}\right), j=1,2, \mathrm{~L}\right\}$. The filter output corresponding to the $j$ th frequency pair can be given by

$$
y_{j}=\frac{\left|\mathbf{v}\left(\phi_{j}^{\prime}, \psi_{j}^{\prime}\right)^{H} \hat{\mathbf{R}}^{-1}{ }_{c-D A-G I P} \mathbf{x}_{0}\right|^{2}}{\mathbf{v}\left(\phi_{j}^{\prime}, \psi_{j}^{\prime}\right)^{H} \hat{\mathbf{R}}^{-1}{ }_{c-D A-G I P \mathbf{v}}\left(\phi_{j}^{\prime}, \psi_{j}^{\prime}\right)} .
$$

Then, we can compare $y_{j}$ with the threshold $\eta$ to perform the final target detection.

\section{Simulation results and discussion}

In the simulations, the main parameters are as follows: the number of array elements is $N=6$, the number of pulses in a CPI is $M=6$. The carrier frequency is $10 \mathrm{GHz}$, and the PRF is $f_{r}=600 H z$. There are 180 clutter patches uniformly distributed in the forward area of antenna array. The noise power is normalized to $0 \mathrm{~dB}$, and the clutter-to-noise ratio (CNR) is $40 \mathrm{~dB}$. It is assumed that a target is located at angle $16.5^{\circ}$, and its normalized Doppler frequency is -0 . 27 . The power of the target is assumed to $10 \mathrm{~dB}$. The resolution scales of the angle-Doppler plane are set as $\rho_{s}=10$ and $\rho_{t}=10$ to achieve a high-resolution power spectrum. Note that the noise power is set to $0 \mathrm{~dB}$ in the simulations. 


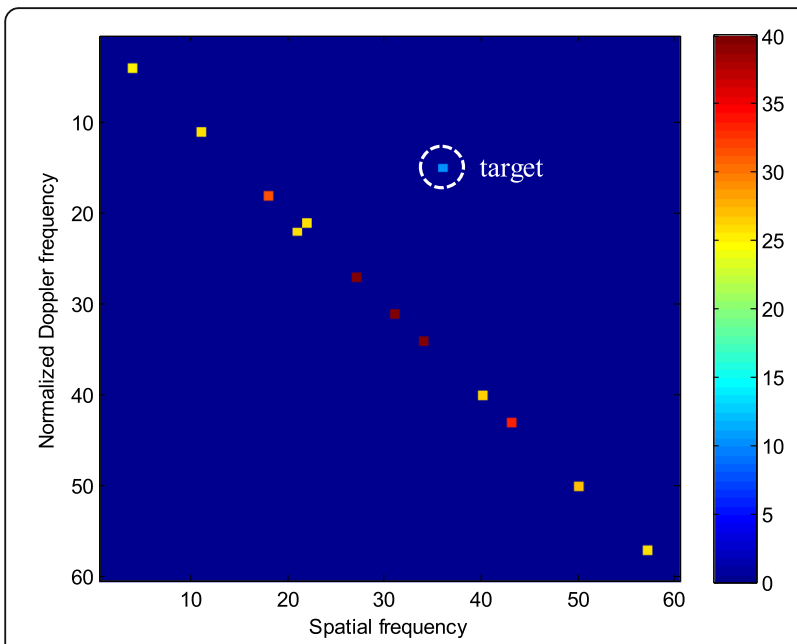

Fig. 5 SR angle-Doppler power spectrum of CUT data without the effect of ICM and knowledge error (dB)

Without loss of generality, the error threshold for the experiment is then set to $\xi=0.1$ which is slightly larger than the noise power.

\subsection{Experiment without ICM and knowledge error}

Firstly, we carry out the experiments without the effect of ICM and knowledge error to verify the effectiveness of the proposed DA-SDS-SRSTAP method. The SR angle-Doppler power spectrum of the CUT data without ICM and knowledge error is presented in Fig. 5. It is clear that the clutters and target are all revealed in the power spectrum, and almost all of the clutter components are located in the clutter ridge, as is analyzed in Section 3. By making the DA-GIP algorithm work on the SR angle-Doppler power spectrum, the DA-GIP values and error values corresponding to four iterative times are shown in Fig. 6. We can see that the DAGIP values have no change from the second iterative time for the reason that the clutter components are mainly located in the clutter ridge, which is consistent with the result shown in Fig. 5. Hence, the iteration should stop at the third time when the error value is lower than the threshold, and the third candidate CCM estimation $\hat{\mathbf{R}}_{c, 3}$ can be applied for the following filter processing. Furthermore, in contrast with the real CCM, the eigenvalues of the candidate CCM estimation of each iterative time are presented in Fig. 7. It is observed that the three groups of eigenvalues are overlapped completely since iteration 2 , and the number of eigenvalues in any of the three groups is nearly equivalent to that of the real CCM. It further demonstrates that the selected CCM estimation can be a good approximation to the real CCM. The normalized filter output with $\hat{\mathbf{R}}_{c, 3}$ is shown in Fig. 8. It is clear that the clutters are completely suppressed and the target is highlighted.

\subsection{Experiment with ICM}

Next, we test the DA-SDS-SRSTAP method in the case of ICM. The SR angle-Doppler power spectrum of the CUT data with ICM is presented in Fig. 9. It is clear that the clutters spread out of the clutter ridge caused by the effect of ICM. The DA-GIP values and error values corresponding to seven iterative times are shown in Fig. 10. Since the clutter extension, the number of iterative time has risen. The iteration should stop at the sixth time when the error value is lower than the threshold, and
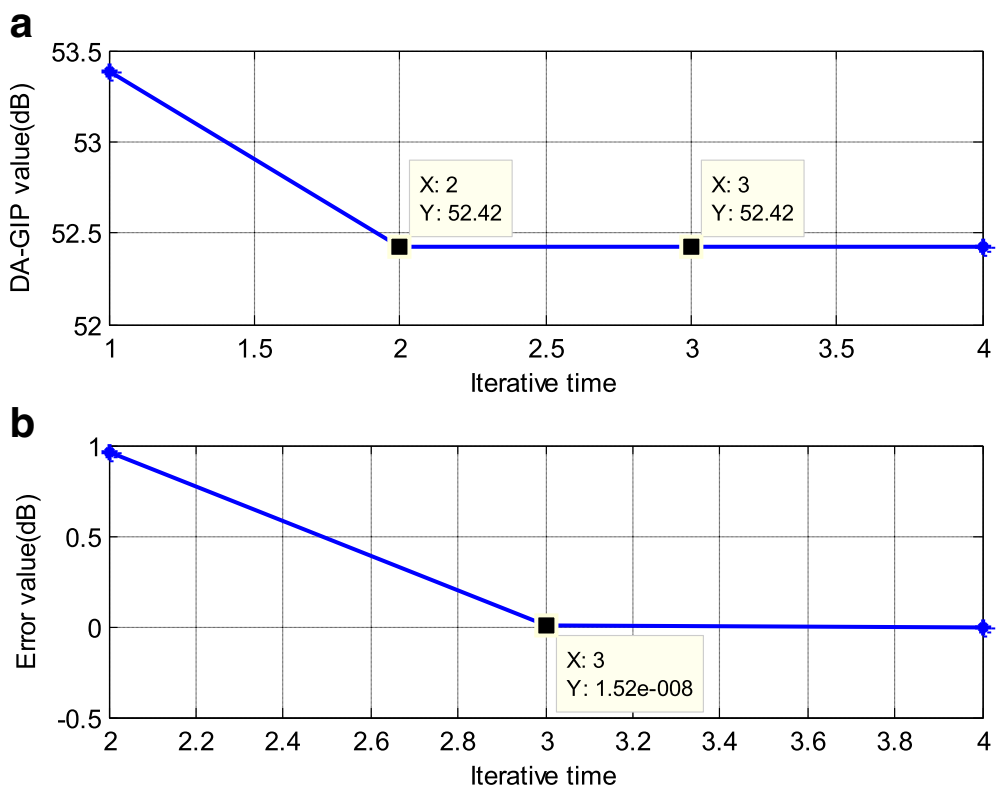

Fig. 6 DA-GIP values and error values without the effect of ICM and knowledge error. a DA-GIP values. b Error values 


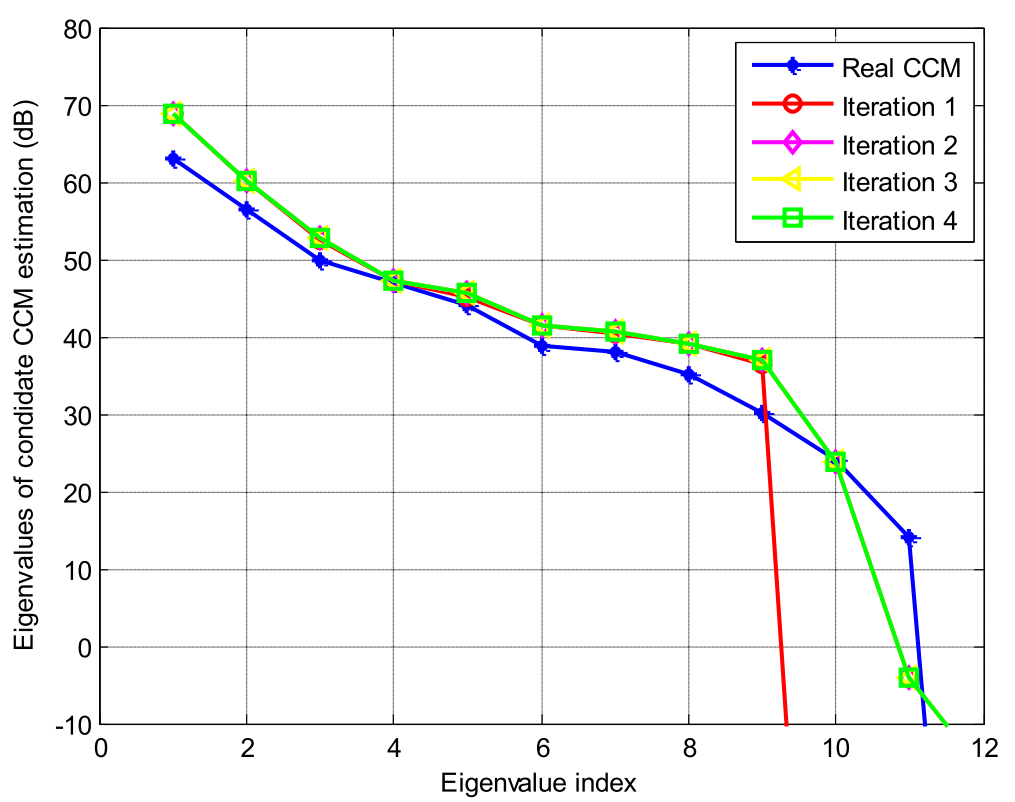

Fig. 7 Eigenvalues corresponding to different CCM estimations without the effect of ICM and knowledge error. $\longrightarrow$ real CCM, $\longrightarrow$ iteration $1, \longrightarrow$ iteration 2, $\rightarrow$ iteration $3, \square-$ iteration 4

the sixth candidate CCM estimation $\hat{\mathbf{R}}_{c, 6}$ can be applied for the following filter processing. Moreover, the eigenvalues of those candidate CCM estimations are also presented in Fig. 11. The eigenvalues are overlapped together since iteration 5 , and the number of eigenvalues of $\hat{\mathbf{R}}_{c, 6}$ is almost the same as the real CCM. Finally, the normalized filter output in the case of ICM is shown in
Fig. 12. We can still see that the clutters are completely suppressed and the target is detected.

\subsection{Detection along range and output SINR}

At last, the target detection along range cell is analyzed, and 100 Monte Carlo simulations are carried out to get an average performance. A number of snapshots from 100

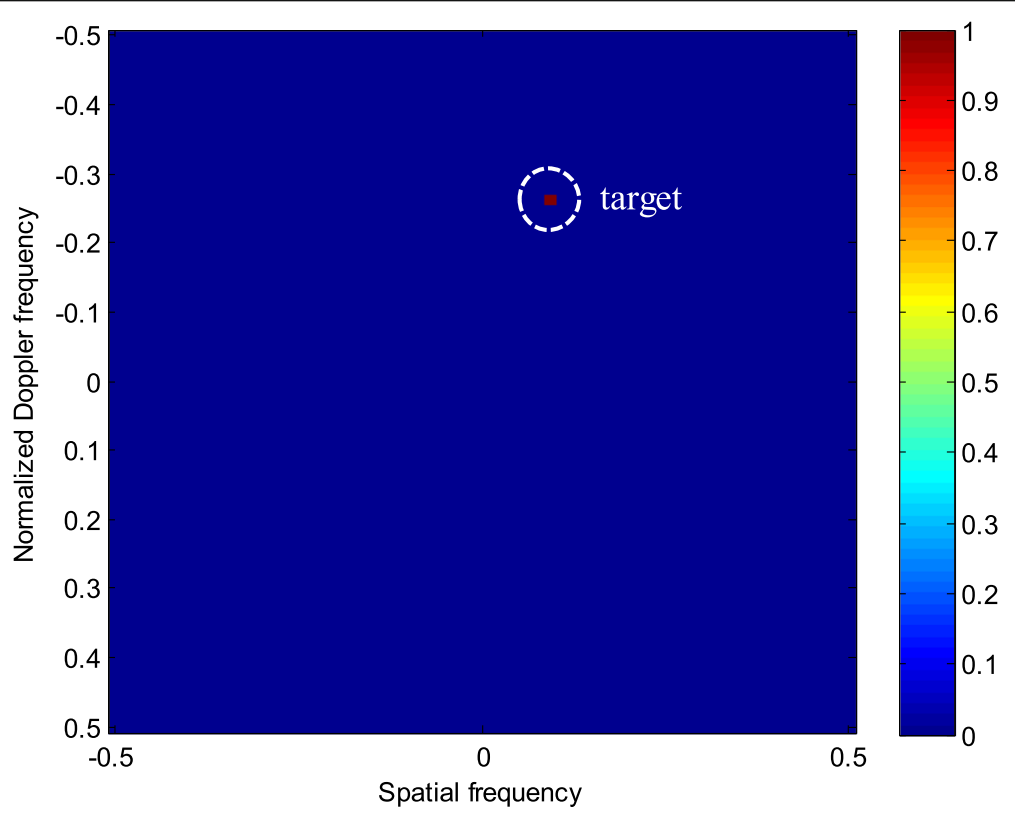

Fig. 8 Normalized STAP filter output without the effect of ICM and knowledge error 


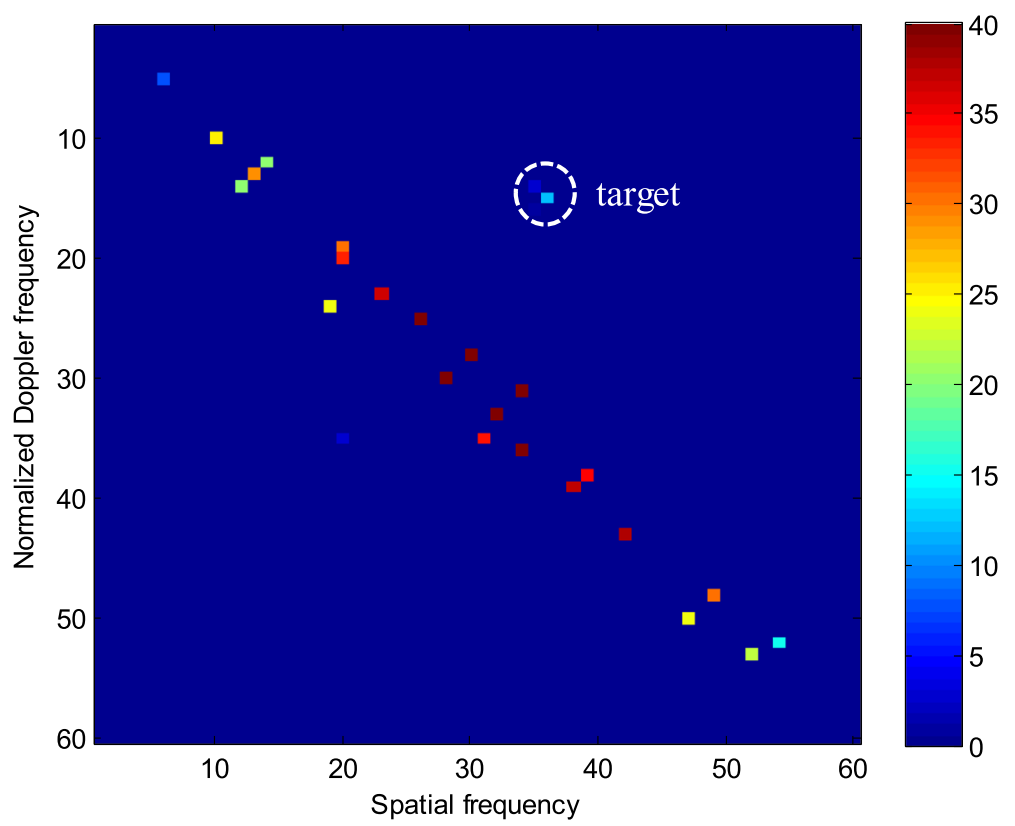

Fig. 9 SR angle-Doppler power spectrum of CUT data with ICM

range cells are processed by D3-STAP, D3SR-STAP, and the proposed DA-SDS-SRSTAP, respectively. The target is assumed to be in the 50th range cell. The detection results along range cell, the output SINR curves, and the probability of detection (Pd) curves versus signal to clutter ratio (SCR) are presented in Figs. 13, 14, and 15, respectively. The probability of false alarm (Pfa) is set to $10^{-4}$. As shown in Fig. 13, each of the three algorithms can effectively suppress the clutters and distinguish the target in both non-ICM and ICM case. Nevertheless, in comparison with D3-STAP, D3SR-STAP and DA-SDSSRSTAP have a less clutter residual along the range cell and own a better target detection performance because they have a higher system DOFs to design the adaptive filter. Moreover, as shown in Fig. 14, D3SR-STAP and DA-SDS-SRSTAP can provide a higher SINR level in the
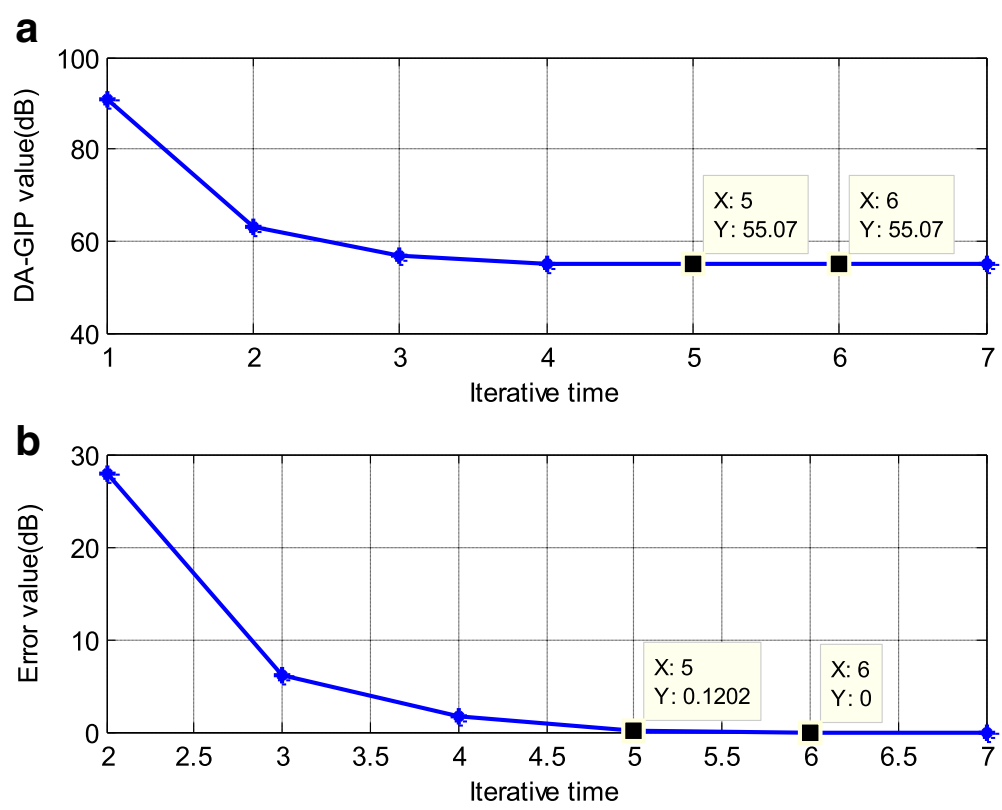

Fig. 10 DA-GIP values and error values with ICM. a DA-GIP values. b Error values 


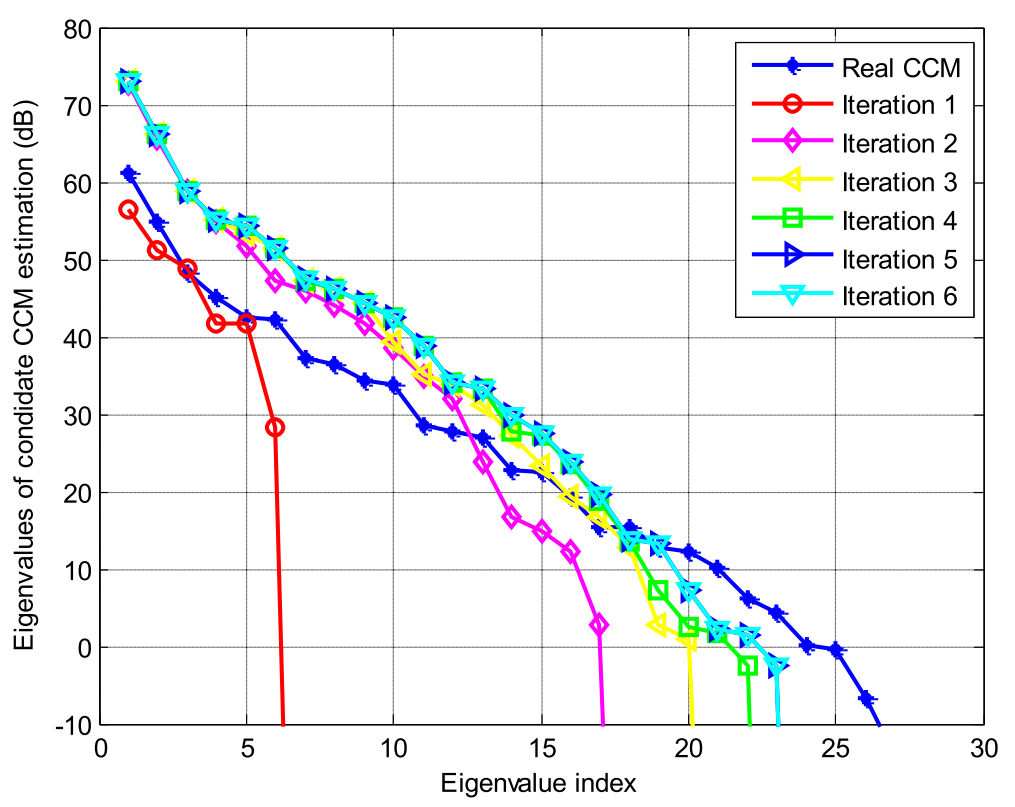

Fig. 11 Eigenvalues corresponding to different CCM estimations with ICM. $\longrightarrow$ real CCM, $\longrightarrow$ iteration $1, \longrightarrow$ - iteration 2, iteration $3,-\mathbf{E}$ iteration $4,-\mathbf{D}$ - iteration $5, \frac{\bar{\nabla}}{-}$ iteration 1

pass-band area and a narrower clutter notch than D3STAP, which means a better performance of minimum detectable velocity (MDV). This improvement is also owing to their characteristics of full system DOFs. Finally, the results shown in Fig. 15 again demonstrate that the detection performances of D3SR-STAP and DA-SDS-SRSTAP are both better than that of D3-STAP due to the loss of DOFs caused by the D3-STAP processing. Furthermore, from Fig. 13 to Fig. 15, we can see that the detection performances without ICM are obviously better than those with ICM. Meanwhile, it is noted that the detection performance of D3-STAP declines more seriously than the ones of D3SR-STAP and DA-SDS-SRSTAP, respectively. Namely, D3SR-STAP and DA-SDS-SRSTAP are more robust to ICM case. One of the main reasons for this can also be owing to the DOFs loss of D3-STAP. Significantly, though D3SR-STAP and DA-SDS-SRSTAP have a similar target detection performance, the DA-SDS-SRSTAP has

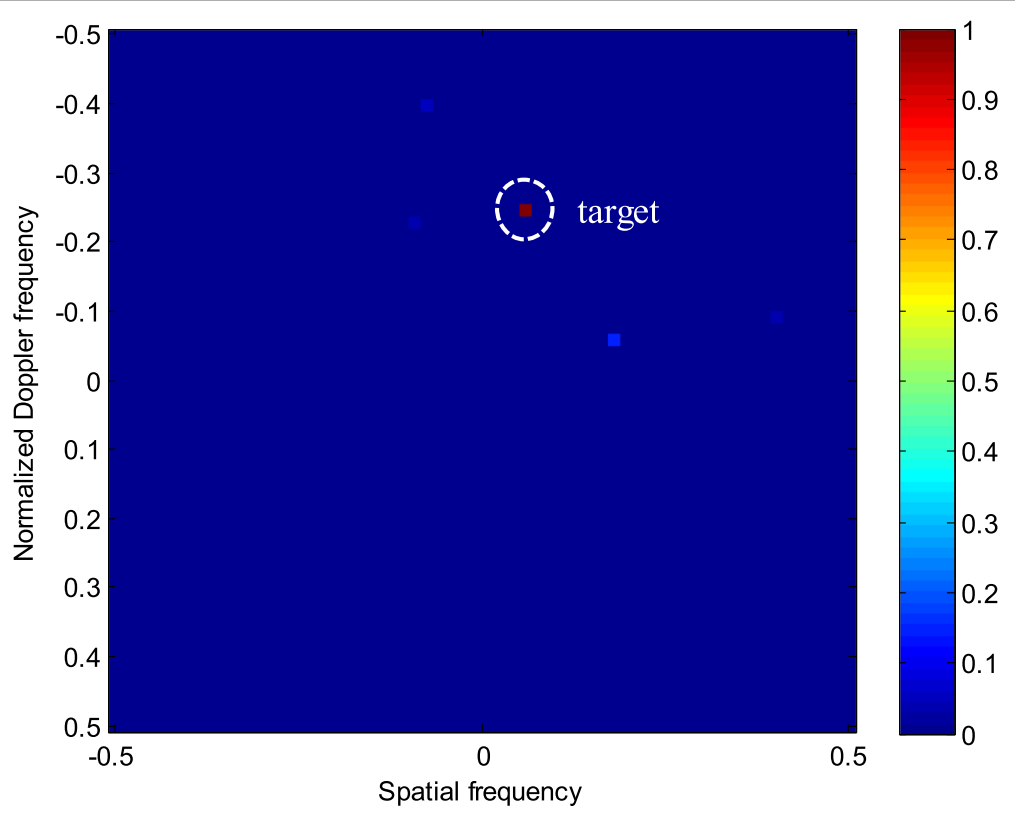

Fig. 12 Normalized STAP filter output in the case of ICM 

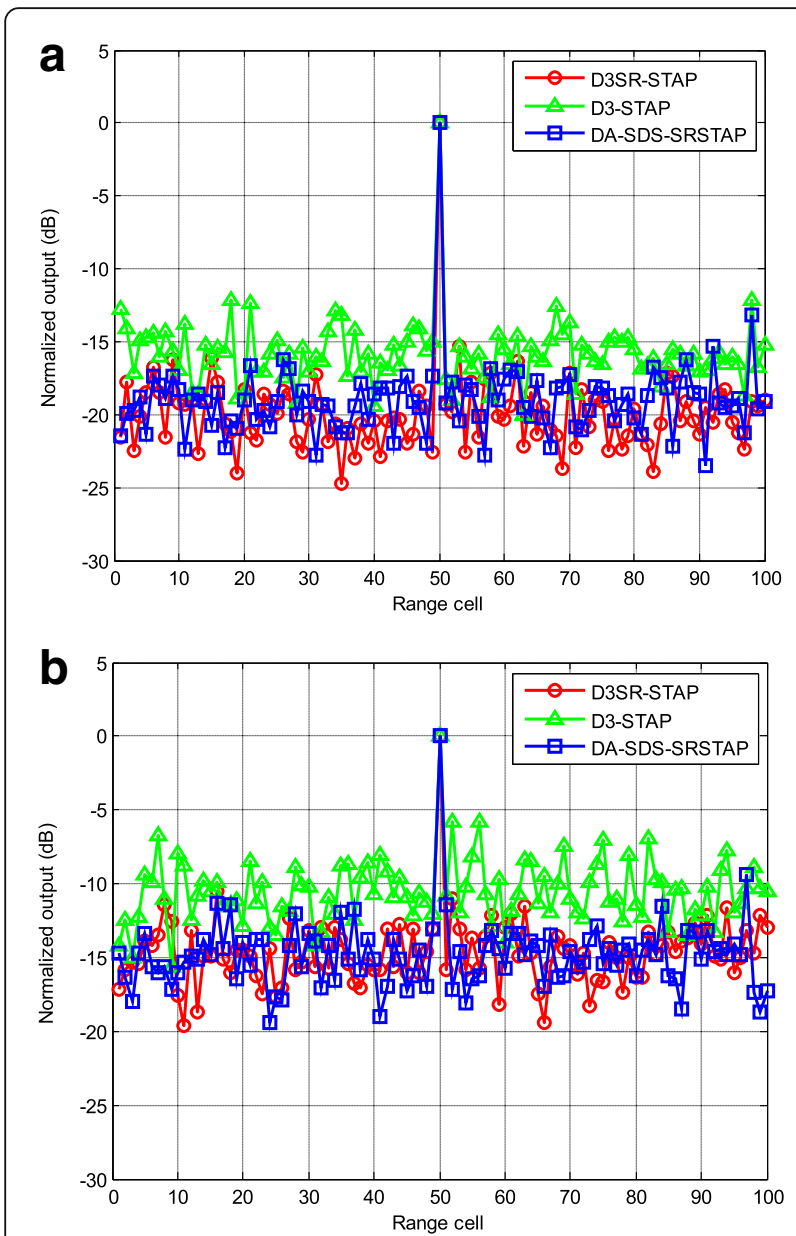

Fig. 13 Normalized STAP filter outputs along range cell. a Without ICM case. b With ICM case. $-\mathbf{6}$ D3SR-STAP, D3-STAP, - DA-SDS-SRSTAP

no use of the prior knowledge of the SOI area that is essential for D3SR-STAP and not easy to be got exactly. Therefore, the DA-SDS-SRSTAP method is more potential to target detection in the non-stationary radar scenario.

\section{Conclusions}

Representative target-free and homogeneous training data are no longer available when facing a severe nonstationary environment (heterogeneous clutter or a high target density), and the performance of classical STAP in this case may deteriorate rapidly. For solving this problem, a novel deterministic-aided single dataset STAP method based on sparse recovery technique (SR) for side-looking airborne or spaceborne radar is proposed in this paper, which is referred to as DA-SDS-SRSTAP. The new method only works with the CUT data, such that its performance is not impacted by the non-stationarity. We exploit the property that the clutter components of sidelooking airborne or spaceborne radar are distributed along
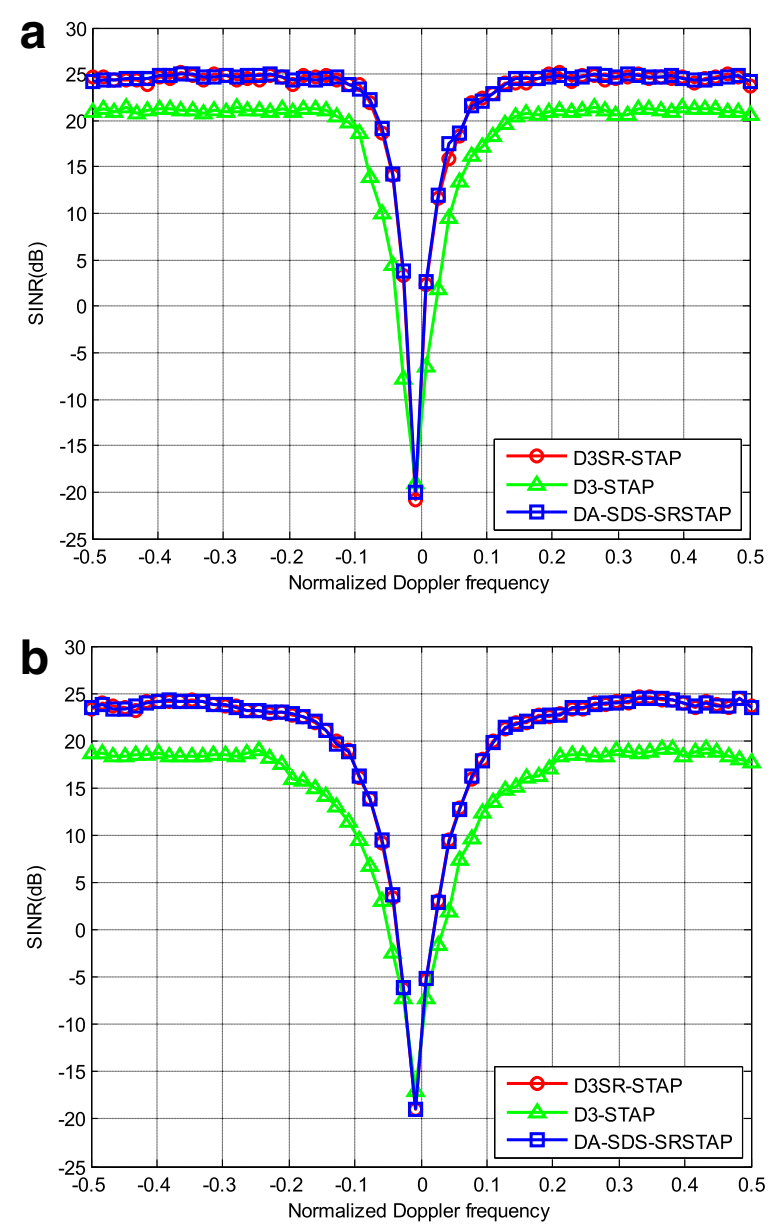

Fig. 14 Output SINR. a Without ICM case. b With ICM case.

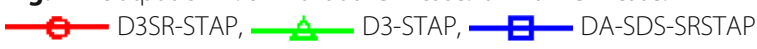

the clutter ridge to perform the estimation of CCM only with the CUT data. We first calculate the high-resolution angle-Doppler power spectrum of CUT data using SR technique. Then, a new adaptive deterministic-aided GIP (DA-GIP) algorithm is presented to recognize and select the clutter components in the SR angle-Doppler power spectrum automatically. Subsequently, the CCM, which is employed to construct the weights of STAP filter, can be estimated by the selected clutter components to fulfill the final STAP filter processing. Firstly, the new method can accomplish the object of target detection without secondary training samples. Secondly, in contrast with the conventional D3-STAP method, the proposed approach can avoid system DOFs loss owing to the usage of SR technique. Thirdly, though the proposed DA-SDS-SRSTAP method and the D3SR-STAP algorithm are both based on the SR technique, the proposed method can detect target without any prior knowledge of the SOI area that is essential for D3SR-STAP and not easy to be got accurately. Consequently, the proposed method has a great potential 

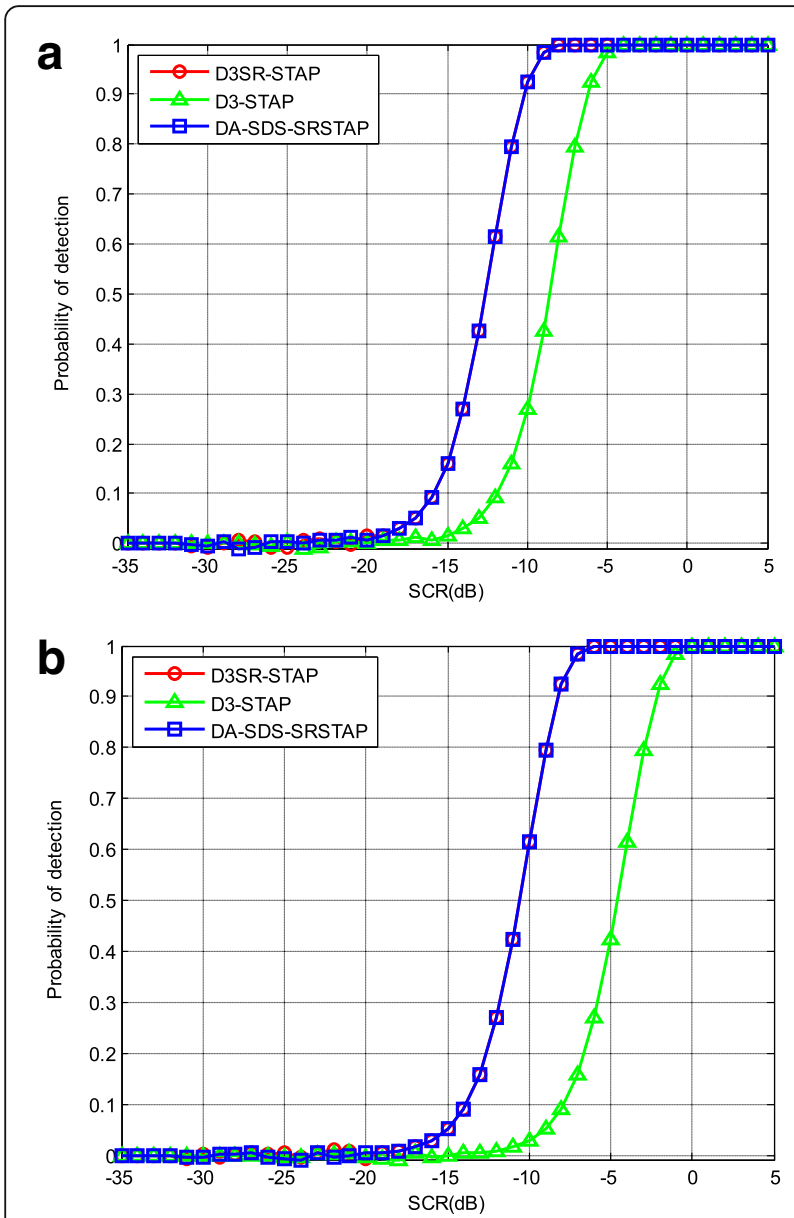

Fig. 15 Probability of detection $(P d)$ curves versus $S C R$, probability of false alarm ( $\mathrm{Pfa}$ ) is set to 10-4. a Without ICM case. b With ICM case. $\mathbf{\square}$ DA-SDS-SRSTAP

for target detection in the heterogeneous clutter environments. Simulation results verify the effectiveness of the proposed detection method. Moreover, how to extend the proposed method to front-looking airborne radar or frequency diverse array (FDA) radar to deal with the rangedependent clutter might be further studied. Though we have just utilized the deterministic knowledge of angleDoppler structure of the clutter to cope with the heterogeneous environments with the help of SR technique, it might be interesting to exploit some other prior knowledges to incorporate with SR technique for improving the STAP performance.

\section{Abbreviations}

CCM: Clutter covariance matrix; CFAR: Constant false alarm rate; CNR: Clutterto-noise ratio; CPI: Coherent processing interval; CUT: Cell under test; D3SRSTAP: Direct data domain space-time adaptive processing using sparse recovery; D3-STAP: Direct data domain space-time adaptive processing: DA: Deterministic aided; DA-GIP: Deterministic-aided generalized inner product; DA-SDS-SRSTAP: Deterministic-aided (DA) single dataset (SDS) space-time adaptive processing (STAP) based on sparse recovery (SR);
DOFs: Degrees of freedom; FDA: Frequency diverse array; FOCUSS: Focal underdetermined system solver; GIP: Generalized inner product; i.i.d: Independent and identically distributed; ICM: Inner clutter motion; KA: Knowledge-aided; MDV: Minimum detectable velocity; MF: Matched filter; MIMO: Multiple-input-multiple-output; MLED: Maximum likelihood estimation detector; NHD: Non-homogeneity detection; Pd: Probability of detection; Pfa: Probability of false alarm; PRF: Pulse repetition frequency; PST: Powerselected training; SCR: Signal to clutter ratio; SDS: Single dataset; SDSSTAP: Single dataset space-time adaptive processing; SINR: Signal-tointerference-plus-noise ratio; SMI: Sample matrix inverse; SOI: Signal of interest; SR: Sparse recovery; SR-STAP: Space-time adaptive processing using sparse recovery; TDS: Two dataset; TDS-STAP: Two dataset space-time adaptive processing; ULA: Uniform linear array

\section{Acknowledgements}

The authors express their gratitude to the editor and anonymous reviewers for their helpful comments and suggestions that improved this paper.

\section{Results section}

Section 4-Simulation results and discussion - of the manuscript can be seen as the combination of the results section and the discussion section.

\section{Methods/experimental section}

This study aims to investigate an effective approach for target detection of airborne/spaceborne radar in heterogeneous clutter environments. We exploit the property of the clutter of side-looking airborne or spaceborne radar and propose a novel deterministic-aided (DA) single dataset (SDS) STAP method based on sparse recovery (SR) technique. We use MATLAB software on computer to generate simulation data for experiment according to the radar parameters described in the manuscript. We make the proposed algorithm work on the simulation data and analyze the corresponding results. Furthermore, we employ Monte Carlo statistical method to test the average performance of target detection along the range bins. All the results have verified the effectiveness of the proposed algorithm.

\section{Availability of data and materials}

The datasets supporting the conclusions of this article are generated using MATLAB software by ourselves according to the radar parameters described in the manuscript.

\section{Authors' contributions}

WW conceived and designed the study, performed the simulations and data analyses, and wrote the manuscript. LZ helped perform the simulations and data analyses with constructive discussions. XW contributed to the conception of the study and manuscript preparation. YY contributed to the conception of the study. All authors reviewed and approved the manuscript.

\section{Authors' information}

Wei Wang is currently a Ph. D. of University of Electronic Science and Technology of China; his research interests include array signal processing, adaptive filter design, and target detection.

Zou Lin is currently an associate professor of University of Electronic Science and Technology of China; his research interests include array signal processing, radar system, and target detection.

Xuegang Wang is currently a professor of University of Electronic Science and Technology of China; his research interests include radar signal processing, radar system, and system simulation.

Yang Yang is currently an engineer of China Aerodynamics Research and Development Center; his research interests include SAR imaging, sonar signal processing, and target detection.

\section{Competing interests}

The authors declare that they have no competing interests.

\section{Publisher's Note}

Springer Nature remains neutral with regard to jurisdictional claims in published maps and institutional affiliations. 
Received: 18 January 2018 Accepted: 11 April 2018

Published online: 24 April 2018

\section{References}

1. WL Melvin, A stap overview. IEEE Aerosp. Electron. Syst. Mag. 19(1), 19-35 (2004)

2. R Klemm, Principles of Space-Time Adaptive Processing (The Institution of Engineering and Technology, London, UK, 2006)

3. CY Chen, PP Vaidyanathan, MIMO radar space-time adaptive processing using prolate spheroidal wave functions. IEEE Trans. Signal Process. 56(2), 623-635 (2008)

4. JR Guerci, Space-Time Adaptive Processing for Radar (Artech House, Boston, 2003)

5. IS Reed, JD Mallet, LE Brennan, Rapid convergence rate in adaptive arrays. IEEE Trans. Aerosp. Electron. Syst. 10(6), 853-863 (1974)

6. FC Robey, DR Fuhrmann, EJ Kelly, R Nitzberg, A CFAR adaptive matched filter detector. IEEE Trans. Aerosp. Electron. Syst. 28(1), 208-216 (1992)

7. Z. Bao, S. Wu, G. S. Liao, and Z. Y. Xu, Review of reduced rank space-time adaptive processing for airborne radar, Proc. Int. Conference on Radar, Beijing, China, 766-769, 1996

8. JR Guerci, JS Goldstein, IS Reed, Optimal and adaptive reduced-rasnk STAP. IEEE Trans. Aerosp. Electron. Syst. 36(2), 647-663 (2000)

9. CD Peckham, AM Haimovich, TF Ayouv, JS Goldstein, IS Reed, Reducedrank STAP performance analysis. IEEE Trans. Aerosp. Electron. Syst. 36(2), 664-676 (2000)

10. XM Li, DZ Feng, HW Liu, D Luo, Dimension-reduced space-time adaptive clutter suppression algorithm based on lower-rank approximation to weight matrix in airborne radar. IEEE Trans. Aerosp. Electron. Syst. 50(1), 53-69 (2014)

11. K Gerlach, Outlier resistant adaptive matched filters. IEEE Trans. Aerosp. Electron. Syst. 38(2), 885-901 (2002)

12. $\mathrm{X}$ Yang, Y Liu, T Long, Robust non-homogeneity detection algorithm based on prolate spheroidal wave functions for space-time adaptive processing. IET Radar Sonar Navig. 7(1), 47-54 (2013)

13. B. Tang, J. Tang, and Y. Peng, Detection of heterogeneous samples based on loaded generalized inner product method Digital Signal Process., 22(4), 605-613, 2012.

14. A Aubry, AD Maio, L Pallotta, A Farina, Median matrices and their application to radar training data selection. IET Radar Sonar Navigation 8(4), 265-274 (2014)

15. A Aubry, AD Maio, L Pallotta, A Farina, Covariance matrix estimation via geometric barycenters and its application to radar training data selection. IET Radar Sonar Navigation 7(6), 600-614 (2013)

16. CT Capraro, GT Capraro, I Bradaric, MC Wicks, WJ Baldygo, Implementing digital terrain data in knowledge-aided space-time adaptive processing. IEEE Trans. Aerosp. Electron. Syst. 42(3), 1080-1099 (2006)

17. AD Maio, D Orlando, C Hao, G Foglia, Adaptive detection of point-like targets in spectrally symmetric interference. IEEE Trans. Signal Process. 64(12), 3207-3220 (2016)

18. AD Maio, D Orlando, An invariant approach to adaptive radar detection under covariance persymmetry. IEEE Trans. Signal Process. 63(5), 1297-1309 (2015)

19. C Hao, D Orlando, G Foglia, G Giunta, Knowledge-based adaptive detection: joint exploitation of clutter and system symmetry properties. IEEE Signal Processing Letters 23(10), 1489-1493 (2016)

20. C Hao, D Orlando, X Ma, C Hou, Persymmetric Rao and Wald tests for partially homogeneous environment. IEEE Signal Processing Letters 19(9), 587-590 (2012)

21. S Bidon, O Besson, JY Tourneret, Knowledge-aided STAP in heterogeneous clutter using a hierarchical Bayesian algorithm. IEEE Trans. Aerosp. Electron. Syst. 47(3), 1863-1879 (2011)

22. $\mathrm{X} Z \mathrm{Zhu}, \mathrm{J}$ Li, P Stoica, Knowledge-aided space-time adaptive processing. IEEE Trans. Aerosp. Electron. Syst. 47(2), 1325-1336 (2011)

23. A Aubry, V Carotenuto, AD Maio, G Foglia, Exploiting multiple a priori spectral models for adaptive radar detection. IET Radar, Sonar Navigation 8(7), 695-707 (2014)

24. V Carotenuto, AD Maio, D Orlando, L Pallotta, Adaptive radar detection using two sets of training data. IEEE Trans. Signal Process. 66(7), 1791, 2018-1801

25. A Aubry, AD Maio, L Pallotta, A geometric approach to covariance matrix estimation and its applications to radar problems. IEEE Trans. Signal Process. 66(4), 907-922 (2018)
26. SM Karbasi, A Aubry, V Carotenuto, MM Naghsh, MH Bastani, Knowledgebased design of space-time transmit code and receive filter for a multipleinput-multiple-output radar in signal-dependent interference. IET Radar, Sonar Navigation 9(8), 1124-1135 (2015)

27. G Cui, X Yu, V Carotenuto, L Kong, Space-time transmit code and receive filter design for colocated MIMO radar. IEEE Trans. Signal Process. 65(5), 1116-1129 (2017)

28. Z Yang, X Li, H Wang, W Jiang, On clutter sparsity analysis in space-time adaptive processing airborne radar. IEEE Geosci. Remote Sens. Lett. 10(5), 1214-1218 (2013)

29. S Sen, Low-rank matrix decomposition and space-time sparse recovery for STAP radar. IEEE J. Sel. Topics Signal Process 9(8), 1510-1523 (2015)

30. S Sen, OFDM radar space-time adaptive processing by exploiting spacetime sparsity. IEEE Trans. Signal Process. 61(1), 118-130 (2013)

31. ZC Yang, X Li, HQ Wang, R Fa, Knowledge-aided STAP with sparserecovery by exploiting spatio-temporal sparsity. IET Signal Processing 10(2), 150-161 (2016)

32. K Sun, H Meng, Y Wang, et al., Direct data domain STAP using sparse representation of clutter spectrum. Signal Process. 91(9), 2222-2236 (2011)

33. TK Sarkar, H Wang, S Park, J Koh, R Adve, K Kim, Y Zhang, MC Wicks, RD Brown, $A$ deterministic least squares approach to space time adaptive processing (STAP). IEEE Trans. Antennas Propag. 49(1), 91-103 (2001)

34. W Choi, TK Sarkar, W Hong, EL Mokole, Adaptive processing using real weights based on a direct data domain least squares approach. IEEE Trans. Antennas Propag. 54(1), 182-191 (2006)

35. D Cristallini, W Bürger, A Robust Direct, Data domain approach for STAP. IEEE Trans. Signal Process. 60(3), 1283-1294 (2012)

36. E. Aboutanios and B. Mulgrew, Evaluation of the single and two data set STAP detection algorithms using measured data, IEEE Int. Geoscience Remote Sensing Symposium, Barcelona, Spain, 494-498, 2007.

37. JF Degurse, L Savy, S Marcos, Reduced-rank STAP for target detection in heterogeneous environments. IEEE Trans. Aerospace Electronic Syst. s 50(2), 1153-1162 (2014)

\section{Submit your manuscript to a SpringerOpen ${ }^{\circ}$ journal and benefit from:}

- Convenient online submission

- Rigorous peer review

- Open access: articles freely available online

- High visibility within the field

- Retaining the copyright to your article

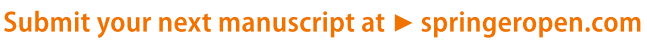

